\title{
Inexistência de Diferenças de Sexo no Fator $g$ (Inteligência Geral) e nas Habilidades Específicas em Crianças de Duas Capitais Brasileiras
}

\author{
There are no Sex Differences on $g$ Factor and Specific Abilities in Children \\ from Two Brazilian Capitals
}

\author{
Carmen E. Flores-Mendoza* ${ }^{a}$, Marcela Mansur-Alves ${ }^{a}$, Álvaro José Lelé ${ }^{b}$ \\ $\&$ Denise Ruschel Bandeira ${ }^{c}$ \\ ${ }^{a}$ Universidade Federal de Minas Gerais - LADI, Belo Horizonte, Brasil \\ ${ }^{b}$ Centro Universitário Newton Paiva - LADI, Belo Horizonte, Brasil \\ ${ }^{c}$ Universidade Federal de Rio Grande do Sul, Porto Alegre, Brasil
}

\begin{abstract}
Resumo
O presente trabalho pretendeu examinar diferenças relacionadas ao sexo no fator $g$ (inteligência geral) e em habilidades específicas em crianças de duas cidades brasileiras: Belo Horizonte e Porto Alegre. No caso da amostra mineira $(n=1,316)$, a idade compreendeu entre 5 e 11 anos de idade examinada através de uma bateria de testes a qual incluiu instrumentos de inteligência (Raven, Escala Verbal WISC III, R-2, DFH), de avaliação psicomotora (Bender) e de desempenho escolar (TDE). No caso da amostra gaúcha ( $n=779$ ), a idade compreendeu entre 5 e 11 anos, utilizando-se o teste Raven - Escala Colorida. A análise individual dos testes aponta que a maioria das diferenças nas habilidades específicas não é estatisticamente significativa. Na utilização do Método dos Vetores Correlacionados, uma análise mais sofisticada da inteligência geral, observa-se ínexistência de diferenças de sexo. Tais resultados corroboram os registros internacionais sobre inexistência de diferenças cognitivas relacionadas ao sexo em crianças e adolescentes.

Palauras-chave: Diferenças de sexo; habilidades específicas; fator g; crianças.
\end{abstract}

\begin{abstract}
This paper intended to verify sex differences on the $g$ factor (general intelligence) and specific abilities from a sample of children from two Brazilian cities: Belo Horizonte (BH) and Porto Alegre (PA). The sample from $\mathrm{BH}(n=1.316)$ aged 5 to 11 years was examined with a battery of intelligence (CPM, Verbal Scale of WISCIII, R-2), psychomotor (Bender) and school achievement tests. The sample from PA $(n=779)$ aged 5 to 11 years was examined with Matrices Progressives Raven. The analysis from these tests shows that the majority of sex differences on specific abilities are not statistically significant. The use of Method Correlated Vectors, which is a most sophisticated method of analyzing general intelligence ( $g$ factor), indicates that there are no sex differences. These results support the international records about non-existence of cognitive difference relate to sex in childhood and adolescence.

Keywords: Sex differences; specific abilities; $g$ factor, children.
\end{abstract}

Há, na atualidade, poucos temas que envolvem tantos debates acadêmicos e sociais como a existência de diferenças de sexo nas habilidades cognitivas. Embora haja um renovado esforço da comunidade científica em esclarecer tal assunto, a resposta definitiva está longe de ser alcançada. $\mathrm{Na}$ literatura observa-se o registro sistemático que homens e mulheres diferem em sua capacidade de processar conteúdos cognitivos. Assim, muitos estudos sugerem que

\footnotetext{
* Endereço para correspondência: Universidade Federal de Minas Gerais, FAFICH - Depto. de Psicologia, Gab. 404,2, Laboratório de Avaliação das Diferenças Individuais (LADI), Av. Antônio Carlos, 6627, Belo Horizonte, MG, $312709-901$. Tel.: (03 1) 3499-6277. E-mail: carmencita@fafich.ufmg.br O presente trabalho recebeu apoio da Fundação de Amparo à Pesquisa do Estado de Minas Gerais (FAPEMIG - Processo $n^{\circ}$ SHA 80257/03) e da Casa do Psicólogo.
}

as mulheres têm, em média, melhor desempenho em tarefas que requerem rápido acesso e uso de informação semântica e fonológica, velocidade perceptual e memória verbal. Os homens, ao contrário, obtêm melhor desempenho em tarefas que exigem raciocínio matemático e científico, orientação espacial e transformações viso-espaciais na memória de trabalho (Codorniu-Roga \& Vigil-Colet, 2003; Halpern, 1997; Lubinsk, 2004; Lynn, Raine, Venables, Mednick, \& Irwing, 2005).

Não obstante, o mesmo consenso não pode ser encontrado quando se muda o foco para as habilidades cognitivas gerais. Lubinsk (2004) define a inteligência geral, ou capacidade cognitiva geral, como uma capacidade mental que envolve, entre outras coisas, a habilidade de raciocinar, planejar, resolver problemas, pensar abstratamente, aprender com a experiência, sendo, portanto, um construto 
extremamente relacionado ao alcance de conquistas acadêmicas, pessoais e profissionais entre outras coisas. Os dados referentes às diferenças de sexo em inteligência geral têm sido acumulados num ritmo extraordinariamente rápido nos últimos dez anos e duas posições principais têm emergido desses resultados. A primeira e, também a mais tradicional, tem afirmado que não há diferenças de sexo em inteligência geral e que quando as mesmas são encontradas são pouco significativas ou decorrentes de problemas metodológicos (para revisão ver Aluja-Fabregat, Colom, Abad \& Juan- Espinosa, 2000; Codorniu-Roga \& Vigil-Colet, 2003; Mackintosh, 1996). No entanto, essa visão tem sido desafiada por Richard Lynn quem propõe uma teoria desenvolvimental para explicar a existência de diferenças de sexo em inteligência (Lynn, 1999). De acordo com essa teoria, meninos e meninas apresentam taxas diferentes de amadurecimento físico e mental durante a infância e a adolescência. Assim sendo, dos nove aos 14 anos há uma aceleração da taxa de crescimento das meninas, porém a partir dos 15 anos o crescimento das meninas desacelera enquanto que o dos meninos continua. Esse princípio geral de diferenças de sexo relacionadas à taxa de maturação aplicar-se-ia ao desenvolvimento de todas as habilidades cognitivas. Seguindo o mesmo raciocínio, não haveria, pois, diferenças de sexo dos seis aos 9 anos, uma vez que nessa faixa etária meninas e meninos desenvolvem-se no mesmo ritmo (Colom \& Lynn, 2004; Lynn, 1999, 2002; Lynn \& Irwing, 2004). Os resultados de estudos em crianças e adolescentes conduzidos em vários países e utilizando os mais consolidados instrumentos de avaliação da inteligência, parecem dar suporte à teoria proposta por Lynn (Lynn, 2002; Lynn, Allik, Pullman, \& Laidra, 2004; Lynn, Backhoff, \& Contreras-Niño, 2004; Lynn, Fergusso, \& Horwood, 2005).

Numa tentativa de aglutinar um grande número de estudos e fortalecer ainda mais a sua posição, Lynn e Irwing (2004) realizaram uma meta-análise de 57 estudos realizados tanto em países desenvolvidos quanto em países em desenvolvimento. Esses estudos referiam-se a três tipos de amostras nos quais utilizaram-se diferentes escalas das Matrizes Progressivas de Raven: (a) diferenças de sexo na população geral [Escalas Geral e Avançada], (b) diferenças de sexo em estudantes universitários [Escala Avançada] e, (c) diferenças de sexo em crianças [Escala Colorida]. Os resultados encontrados na população geral (Escala Geral) apontaram não haver diferenças de sexo entre crianças de 6 a 14 anos de idade, mas os meninos obtêm médias mais elevadas a partir dos 15 anos. Entre os adultos, a vantagem masculina eqüivale a 5 pontos de QI. Ao considerar apenas os estudos dos países em desenvolvimento que utilizaram o Raven Geral, os meninos de 9 e 10 anos apresentaram médias mais altas. Depois, aos 11 e 12 anos, foram as meninas que apresentaram um escore superior. Contudo, a partir dos 14 anos de idade, novamente os meninos apresentaram uma pontuação maior. Esses resultados confirmam o pressuposto de Lynn citado anteriormente, ou seja, parece haver uma vantagem a favor dos homens somente a partir dos 15 anos. Embora pareça haver suporte para a posição defendida por Lynn, vindo também de medidas de eficiência neuronal (Neubauer \& Fink, 2003), o panorama encontrado na infância é, ainda, muito difuso. Recentemente, Abdel-Kalek e Lynn (2006) apresentaram dados de aplicação do teste Raven Escala Geral a uma amostra ampla de crianças e adolescentes do Kuwait $(n=6.529)$. Também apresentaram dados da aplicação de testes educacionais (compreensão verbal, aprendizagem de língua estrangeira e matemática). Os resultados do teste Raven são favoráveis às meninas, com um ganho de 1,2 pontos de QI com relação aos meninos. Nos testes educacionais também as meninas têm desempenho significativamente superior.

Outro ponto de discordância acerca de diferenças de gênero é a necessidade de estabelecer-se uma diferença conceitual bastante importante: instrumentos como as escalas Wechsler ou o teste Raven, por exemplo, são apenas veículos de construtos que se expressam em medidas (ex. QI). Esses veículos carregariam a inteligência em geral (e com eles inteligência geral + habilidades específicas). Com o intuito de identificar um fator $g$ de forma mais pura e, portanto, extrair com maior precisão a inteligência geral, os investigadores utilizam o Método dos Vetores Correlacionados (MVC) proposto por Jensen (1998). São poucos os estudos envolvendo esse método. Recentemente, Nyborg (2005) apresentou um estudo de MVC com dados de um conjunto variado de testes cognitivos em que obteve diferenças favoráveis aos homens $(3,8$ pontos de QI). Esses resultados, portanto, contrariam o estudo de Colom, Juan-Espinosa, Abad e Garcia (2000) realizado em uma larga amostra $(N=10.475)$ com o WAIS III, segundo o qual não haveriam diferenças significativas no fator $g$ relacionadas ao sexo. Mas, Nyborg alega que o estudo de Colom et al. (2000) apresenta problemas de metodologia, pois os autores ao combinar diferentes cargas fatoriais da variável sexo sobre $g$ provocaram que alguns valores eliminassem outros. O produto seria uma diferença aparentemente «nula» entre os sexos na inteligência geral (ou fator $g$ ). No entanto, uma cuidadosa observação da metodologia utilizada no próprio estudo de Nyborg permite verificar um procedimento estranho a qualquer trabalho psicométrico: a realização de uma análise fatorial de numerosos testes $(n=20)$ aplicados a uma amostra extremamente pequena (31 para cada sexo). Por outro lado, se desconhece na literatura internacional qualquer aplicação do MVC em dados de crianças, muito menos sobre dados de países em desenvolvimento, como o Brasil.

Nesse sentido, o presente estudo objetivou analisar os dados sistematicamente coletados pelo Laboratório de Avaliação das Diferenças Individuais (LADI) desde 2002 e efetivar estudos sobre diferenças de sexo em crianças da cidade Belo Horizonte, os quais envolveram não só medidas de inteligência, mas também de desenvolvimento psicomotor e de desempenho escolar. Também, por ocasião da recente normatização da Escala Colorida de Raven para Porto Alegre, o estudo estendeu-se a esses 
dados. Duas estratégias de análise foram utilizadas: índices $d$, obtidos da aplicação de testes individuais, e MVC, aplicado a resultados de uma bateria de testes cognitivos.

\section{Método}

\section{Amostra}

Participaram do estudo diversas amostras de crianças de faixa etária entre cinco e 11 anos de idade, regularmente matriculadas em escolas da rede pública e particular de ensino das cidades de Belo Horizonte (MG) e de Porto Alegre (RS). Assim sendo, o número de participantes variou de acordo com o instrumento utilizado para a coleta dos dados e de acordo com a procedência geográfica. No caso de Belo Horizonte, participaram um total de 1.316 crianças entre cinco e 11 anos de idade. Em um primeiro momento, foram avaliadas 335 crianças $(171$ meninos e 164 meninas) com o teste Raven Escala Colorida, o Desenho da Figura Humana (DFH) e a Escala Verbal do WISC III. Em um segundo momento avaliaram-se 456 crianças com o Teste de Desempenho Escolar-TDE (236 meninos e 220 meninas). Em um terceiro momento avaliaram-se 525 crianças com o teste não-verbal de inteligência R-2 (279 meninos e 246 meninas) e uma parte dela com o teste guestáltico viso-motor de Bender $(n=511 ; 276$ meninos e 235 meninas) e com o Desenho da Figura Humana ( $n=376$; 211 meninos e 165 meninas). Na amostra de Porto Alegre, foi avaliado um total de 779 crianças entre 4 anos e 9 meses e 11 anos e 8 meses de idade, sendo 393 meninas e 386 meninos.

$\mathrm{Na}$ amostra mineira, os testes Raven Escala Colorida, DFH e Escala Verbal do WISC III foram administrados durante o ano de 2002 e o TDE no início de 2004. Esses instrumentos foram aplicados em uma escola pública. $\mathrm{Na}$ extensão do estudo, os testes Bender, DFH e R-2 foram aplicados no segundo semestre de 2004 e início de 2005 em amostras de crianças provenientes de seis instituições de ensino, sendo três públicas e três particulares. Na amostra gaúcha, o teste Raven Escala Colorida foi aplicado entre os anos de 1995 a 2003, em escolas públicas sorteadas aleatoriamente.

\section{Instrumentos}

Utilizaram-se os seguintes instrumentos psicológicos:

1. Testes de inteligência: Teste das Matrizes Progressivas de Raven-Escala Colorida (Angelini, Alves, Custódio, Duarte \& Duarte, 1999); Escala Verbal do WISC III (Figueiredo, 2002); teste R2 (Oliveira, Rosa \& Alves, 2000) e o teste Desenho da Figura Humana (Wechsler, 2003).

2. Teste Psicomotor: Bender (Koppitz, 1989).

3. Teste psicoeducacional: Teste de Desempenho Escolar-TDE (Stein, 1994).

\section{Procedimento}

$\mathrm{Na}$ amostra de crianças mineiras, as aplicações do Raven Escala Colorida, da Escala Verbal WISC III e do TDE fazem parte de um projeto de avaliação psicológica longi- tudinal de crianças escolares vinculado a um acordo acadêmico-científico entre o LADI/UFMG e a escola pública local de coleta de dados ${ }^{1}$. Para efeito das aplicações dos testes Bender, DFH e R-2, as autoridades educacionais de seis escolas (três públicas e três particulares) de Belo Horizonte foram contatadas, às quais solicitou-se permissão para a realização do estudo.

Os testes Raven Escala Colorida e DFH foram aplicados de forma coletiva a partir dos 9 anos de idade (aos $7 \mathrm{e}$ 8 anos de idade esses testes foram aplicados de forma individual). Os demais instrumentos (Escala Verbal WISC III, R2, Bender e TDE) foram aplicados individualmente, seguindo as instruções contidas nos respectivos manuais.

Todos os participantes foram testados por uma equipe de estudantes de psicologia, integrantes do LADI, devidamente treinados no Laboratório e nas dependências das escolas participantes.

$\mathrm{Na}$ amostra gaúcha, por se tratar de um estudo de normatização do teste Raven Escala Colorida, as escolas foram aleatoriamente sorteadas, assim como os participantes, respeitando o critério de duas crianças de cada sexo por faixa etária por escola. O teste Raven Escala Colorida foi aplicado tanto de forma coletiva, em grupos de no máximo oito crianças, como individual, no caso de crianças pré-escolares ou sem condições de registrar as respostas. Assim como no estudo mineiro, todos os participantes foram testados por alunos de psicologia que trabalhavam em pesquisa com uma das autoras.

\section{Técnicas de Análises}

\section{Estimativa do indice d}

O índice $d$, ou diferença sigma $(\sigma$ diff), constitui diferenças de sexo expressas em unidades de desvios padrões. Este índice permite fazer comparações entre resultados vindos da aplicação de diferentes instrumentos de medida psicológica. Para estimá-lo, se identifica a diferença das médias dos grupos e logo se divide o valor obtido pela média dos desvios-padrões (Jensen, 1998, p. 403). A fórmula para tal cálculo é:

$$
d=\frac{X_{\mathrm{a}}-X_{\mathrm{b}}}{\left.\sqrt{ }\left[\left(N_{\mathrm{a}} \sigma_{\mathrm{a}}^{2}+N_{\mathrm{b}} \sigma_{\mathrm{b}}^{2}\right) / N_{\mathrm{a}}+N_{\mathrm{b}}\right)\right]}
$$

Onde X é a média, $a$ e $b$ são os grupos de sexo (ex. $a$ masculino e $b$ feminino), $N$ é o tamanho da amostra de cada grupo de sexo, e $\sigma$ é o desvio padrão. Por convenção acadêmica um valor $d$ negativo indicará resultado a favor das meninas, desde que as médias deste grupo ingressem em segundo lugar.

O índice $d$ foi calculado para os testes DFH, R2, TDE, Bender aplicados na amostra mineira e para o Raven Escala Colorida da amostra gaúcha.

\footnotetext{
${ }^{1}$ Ver maiores detalhes do projeto no site: http:// www.fafich.ufmg.br/ ladi no link Pesquisa.
} 


\section{Método dos vetores correlacionados}

O Método de Vetores Correlacionados (MVC) constitui uma técnica sofisticada criada por Jensen (1998) para comparar os vetores das cargas $g$ de cada teste cognitivo para cada sexo com as diferenças padronizadas das médias dos grupos (índices $d$ ). O MVC é calculado quando se trata de aplicações de diversos testes a uma amostra considerável e representativa de sua população. Para tanto, primeiro se faz necessário que o índice de precisão $\left(\mathrm{r}_{\mathrm{xx}}\right)$ de cada teste seja adequado. Logo, deve-se provar que o fator $g$ extraído de cada teste é o mesmo para ambos os sexos. O índice de congruência serve a essa finalidade. Sua fórmula é:

$$
r_{\mathrm{c}}=\Sigma \mathrm{XY} / \sqrt{ } \Sigma \mathrm{X}^{2} \Sigma \mathrm{Y}^{2}
$$

Onde X representa a carga $g$ de um dos grupos (ex. mulheres) e Y representa a carga $g$ do outro grupo (ex. homens). Se considera similaridade entre os fatores (cargas g) quando se obtém um coeficiente de congruência acima de 0,95. Se obtido, se estabelece posteriormente um vetor $g$ utilizando a média dos vetores $g$ de ambos os sexos. E, finalmente, se calcula a correlação Pearson e Spearman para observar o grau de relação entre os vetores (cargas $g$ e índices $d$ ).

O MVC foi utilizado para os dados provenientes de oito testes cognitivos: DFH, Raven Escala Colorida e os seis subtestes da Escala Verbal do WISC III. Esses testes fo-

Tabela 1

Média, Desvio e Padrão e Valores d em DFH, R-2 e Bender [amostra mineira]

\begin{tabular}{|c|c|c|c|c|c|c|c|c|c|c|}
\hline \multirow[t]{2}{*}{ Teste } & & \multicolumn{3}{|c|}{ 5-7 anos } & \multicolumn{3}{|c|}{ 8-9 anos } & \multicolumn{3}{|c|}{ 10-11 anos } \\
\hline & & $N$ & Média & $D P$ & $N$ & Média & $D P$ & $N$ & Média & $D P$ \\
\hline \multirow[t]{3}{*}{ DFH } & Masculino & 77 & 20,81 & 6,48 & 83 & 30,46 & 7,14 & 51 & 34,14 & 5,55 \\
\hline & Feminino & 62 & 24,27 & 6,35 & 59 & 31,04 & 6,55 & 44 & 34,73 & 5,93 \\
\hline & $d$ & \multicolumn{3}{|c|}{$-0,538(8,07)^{*}$} & \multicolumn{4}{|c|}{$-0,084$} & \multicolumn{2}{|c|}{$-0,103$} \\
\hline \multirow[t]{3}{*}{$\mathrm{R}-2$} & Masculino & 114 & 15,47 & 5,11 & 109 & 20,00 & 5,49 & 56 & 21,86 & 4,18 \\
\hline & Feminino & 93 & 15,54 & 5,36 & 100 & 20,21 & 4,54 & 53 & 22,09 & $4,2 \mathrm{O}$ \\
\hline & $d$ & \multicolumn{3}{|c|}{$-0,013$} & \multicolumn{3}{|c|}{$-0,041$} & & \multicolumn{2}{|c|}{$-0,054$} \\
\hline \multirow[t]{3}{*}{ Bender } & Masculino & 114 & 10,82 & 4,88 & 107 & 5,62 & 3,38 & 55 & 4,42 & 2,90 \\
\hline & Feminino & 90 & 9,80 & 4,07 & 92 & 5,24 & 2,74 & 53 & 4,64 & 2,87 \\
\hline & $d$ & \multicolumn{3}{|c|}{ - 0,224 * } & \multicolumn{3}{|c|}{$-0,122$} & \multicolumn{3}{|c|}{0,086} \\
\hline
\end{tabular}

Nota. No teste psicomotor Bender, o sistema de pontuação é o inverso ao dos testes cognitivos, portanto, uma média menor indica um desempenho melhor; *n. sig. $<0,05$. Entre parêntese o valor $d$, estatisticamente significativo, convertido a pontos de QI. No caso do teste psicomotor Bender, resulta inapropriado converter o valor $d$ a QI.

A Tabela 2 mostra os resultados das diferenças de sexo tanto para o escore total quanto para cada subteste do TDE. Para o escore total, podem ser verificadas diferenças a favor das meninas em todas as séries. Também, nos subtestes de escrita e leitura, as meninas obtêm melhor desempenho do que os meninos em todas as séries escolares sendo apenas estatisticamente significativa a diferença ram aplicados à escola de Ensino Fundamental da UFMG, sendo esta caracterizada pela alta heterogeneidade econômica e diversidade de procedência geográfica das crianças que a freqüentam, razões que permitem considera-la como aproximadamente representativa de Belo Horizonte (Colom \& Flores-Mendoza, 2007).

\section{Resultados}

\section{Índices d de Testes Individuais}

\section{Amostra Mineira}

As tabelas 1 e 2 mostram os escores médios, os desvios padrões de ambos os sexos, os valores $d$ para cada faixa etária. A correspondência em QI do valor $d$ significativo se apresenta entre parêntese (para tanto multiplica-se o valor $d$ por 15 ).

No caso da Tabela 1, são mostrados os resultados das diferenças de sexo no DFH, R-2 e Bender, os dois primeiros testes considerados como medidas cognitivas. No DFH e no R-2, a diferença encontrada favorece as meninas nas três faixas etárias (5-7; 8-9; 10-11 anos). Destaca-se que no DFH, as idades de cinco a sete anos apresentaram um valor $d \mathrm{O}, 54$ (equivalente a 8 pontos de $\mathrm{QI}$ ), o maior valor observado em todo o estudo, sendo também estatisticamente significativo. No Bender (teste psicomotor), as meninas mostram maior desempenho do que os meninos dos cinco aos nove anos, sendo estatisticamente significativa apenas a diferença dos cinco aos sete anos de idade. 
Flores-Mendoza, C. E., Mansur-Alves, M., Lelé, A. J. \& Bandeira, D. R. (2007). Inexistência de Diferenças de Sexo no Fator $g$ (Inteligência Geral) e nas Habilidades Específicas em Crianças de Duas Capitais Brasileiras

Tabela 2

Média, Desvio-Padrão e Valores d no Teste de Desempenho Escolar [amostra mineira]

\begin{tabular}{|c|c|c|c|c|c|c|c|c|c|c|}
\hline \multirow[t]{2}{*}{ Teste } & \multirow{2}{*}{ Sexo } & \multicolumn{3}{|c|}{$1^{o}-2^{o}$} & \multicolumn{3}{|c|}{$3^{o}-4^{o}$} & \multicolumn{3}{|c|}{$5^{\circ}-6^{o}$} \\
\hline & & $N$ & Média & $D P$ & $N$ & Média & $D P$ & $N$ & Média & $D P$ \\
\hline \multirow[t]{3}{*}{ TDE } & \multirow{3}{*}{$\begin{array}{l}\text { Masculino } \\
\text { Feminino } \\
d\end{array}$} & 80 & 85,65 & 12,30 & 78 & 109,99 & 10,33 & 78 & 118,72 & 8,70 \\
\hline & & 82 & 86,77 & 12,80 & 77 & $111, \mathrm{OO}$ & 9,41 & 61 & 120,90 & 8,87 \\
\hline & & & \multicolumn{2}{|c|}{ - 0,089 } & \multicolumn{3}{|c|}{ - 0,102} & \multicolumn{3}{|c|}{$-0,248$} \\
\hline \multirow[t]{3}{*}{ Escrita } & Masculino & 80 & 18,97 & 5,65 & 78 & 27,08 & 4,74 & 78 & 28,63 & 3,73 \\
\hline & Feminino & 82 & 19,49 & 5,60 & 77 & 27,88 & 4,62 & 61 & 30,13 & $4, \mathrm{O} 4$ \\
\hline & $d$ & & \multicolumn{2}{|c|}{ - 0,092 } & \multicolumn{3}{|c|}{$-0,170$} & \multicolumn{3}{|c|}{$-0,387^{*}$} \\
\hline \multirow[t]{3}{*}{ Leitura } & Masculino & 80 & 57,93 & 6,78 & 78 & 65,95 & 4,31 & 78 & 66,99 & 3,09 \\
\hline & Feminino & 82 & 59,49 & 6,31 & 77 & 66,08 & 3,87 & 61 & 67,07 & 5,71 \\
\hline & $d$ & & \multicolumn{2}{|c|}{$-0,238$} & \multicolumn{3}{|c|}{$-0,031$} & \multicolumn{3}{|c|}{$-0,018$} \\
\hline \multirow{3}{*}{$\begin{array}{l}\text { Arit- } \\
\text { mética }\end{array}$} & Masculino & 80 & 8,54 & 2,99 & 78 & 17,10 & 3,47 & 78 & 23,54 & 4,77 \\
\hline & Feminino & 82 & 8,18 & 3,16 & 77 & 17,08 & 3,19 & 61 & 22,64 & 5,97 \\
\hline & $d$ & & \multicolumn{2}{|c|}{0,117} & \multicolumn{3}{|c|}{0,006} & \multicolumn{3}{|c|}{0,168} \\
\hline
\end{tabular}

Nota. *..n.sig. <0,05.

\section{Amostra Gaúcha}

A tabela 3 mostra os escores médios, os desvios padrões de ambos os sexos e os valores $d$ em cada faixa etária. Os resultados no teste Raven mostram não haver diferenças cognitivas significativas relacionadas à variável sexo em nenhuma faixa etária.

Tabela 3

Média, Desvio-Padrão e Valores d no Teste Raven Escala Colorida [amostra gaúcha]

\begin{tabular}{|c|c|c|c|c|c|c|c|c|c|c|c|c|c|}
\hline \multirow[t]{2}{*}{ Teste } & & \multicolumn{3}{|c|}{$4 a 9 m .-6 a .8 m}$. & \multicolumn{3}{|c|}{$6 \mathrm{a} .9 \mathrm{~m} .-8 \mathrm{a} .8 \mathrm{~m}$} & \multicolumn{3}{|c|}{$8 \mathrm{a} .9 \mathrm{~m} .-10 \mathrm{a} .8 \mathrm{~m}}$. & \multicolumn{3}{|c|}{$10 \mathrm{a} .9 \mathrm{~m} .-11 \mathrm{a} .8 \mathrm{~m}$} \\
\hline & & $N$ & Média & $D P$ & $N$ & Média & $D P$ & $N$ & Média & $D P$ & $N$ & Média & $D P$ \\
\hline \multirow{3}{*}{$\begin{array}{l}\text { Raven } \\
\text { Escala } \\
\text { Colorida }\end{array}$} & Masculino & 106 & 16,42 & 3,80 & 103 & 20,41 & 4,99 & 122 & 25,02 & 4,08 & 55 & 27,44 & 4,70 \\
\hline & Feminino & 120 & 16,23 & 3,67 & 112 & 19,84 & 4,37 & 114 & 24,51 & 4,17 & 47 & 27,81 & 4,24 \\
\hline & $d$ & & \multicolumn{2}{|c|}{0,059} & \multicolumn{3}{|c|}{0,121} & \multicolumn{4}{|c|}{0,123} & \multicolumn{2}{|c|}{$-0,08$} \\
\hline
\end{tabular}

\section{Método de Vetores Correlacionados}

Para efeito da aplicação do MVC utilizou-se os resultados de uma amostra de 335 crianças com idade entre 7 e 11 anos, na qual aplicou-se oito testes cognitivos, sendo seis da Escala Verbal WISC III (Informação, Semelhan- ças, Aritmética, Vocabulário, Compreensão, Dígitos), e os testes Raven-Escala Colorida e DFH. As estatísticas descritivas, os índices $d$ e as correlações ponto-bisserial $\left({ }_{r} \mathrm{pb}\right)$ entre sexos e os subtestes cognitivos encontram-se na Tabela 4.

Tabela 4

Estatísticas Descritivas de Cada Subteste, Índices de Correlação Ponto-Bisserial (gênero x subteste)

\begin{tabular}{lllllll}
\hline \multirow{2}{*}{ Testes } & \multicolumn{2}{l}{ Masculino $(n=171)$} & \multicolumn{2}{l}{ Feminino $(n=164)$} & & $\mathrm{r}_{\mathrm{pb}}$ \\
\cline { 2 - 7 } & $M$ & $D P$ & $M$ & $D P$ & $d$ & 0,105 \\
\hline Informação & 13,56 & 4,33 & 12,71 & 3,67 & 0,211 & $-0,076$ \\
Semelhanças & 10,85 & 5,92 & 11,71 & 5,40 & $-0,152$ & 0,032 \\
Aritmética & 15,02 & 2,98 & 14,84 & 2,59 & 0,064 & 0,052 \\
Vocabulário & 23,00 & 6,62 & 22,32 & 6,48 & 0,104 & $-0,018$ \\
Compreensão & 14,40 & 4,77 & 14,57 & 4,61 & $-0,036$ & $-0,072$ \\
Dígitos & 11,19 & 2,95 & 11,60 & 2,71 & $-0,145$ & 0,046 \\
Raven Colorido & 26,53 & 5,70 & 25,98 & 6,11 & 0,093 & $-0,067$ \\
DFH & 30,84 & 7,35 & 31,74 & 5,84 & $-0,135$ & \\
\hline
\end{tabular}


Na Tabela 5 apresenta-se a matriz de correlações entre os testes cognitivos para cada sexo e os índices de preci- são de cada teste. Observa-se que as correlações tanto na amostra masculina quanto na feminina são moderadas a altas.

Tabela 5

Matriz de Correlações entre Subtestes e Coeficientes de Precisão

\begin{tabular}{llllllllll}
\hline Testes & 1 & 2 & 3 & 4 & 5 & 6 & 7 & 8 & $\mathrm{r}_{\mathrm{xx}}$ \\
\hline 1. Informação & $\mathbf{1 . 0}$ & 0,723 & 0,704 & 0,742 & 0,679 & 0,424 & 0,570 & 0,433 & 0,886 \\
2. Semelhanças & 0,772 & $\mathbf{1 , 0}$ & 0,692 & 0,757 & 0,670 & 0,459 & 0,633 & 0,468 & 0,869 \\
3. Aritmética & 0,698 & 0,632 & $\mathbf{1 , 0}$ & 0,589 & 0,626 & 0,489 & 0,594 & 0,453 & 0,815 \\
4. Vocabulário & 0,805 & 0,754 & 0,611 & $\mathbf{1 , 0}$ & 0,682 & 0,372 & 0,618 & 0,454 & 0,869 \\
5. Compreensão & 0,668 & 0,649 & 0,600 & 0,686 & $\mathbf{1 , 0}$ & 0,337 & 0,476 & 0,446 & 0,802 \\
6. Dígitos & 0,503 & 0,487 & 0,579 & 0,442 & 0,403 & $\mathbf{1 , 0}$ & 0,472 & 0,340 & 0,800 \\
7. Raven Colorido & 0,598 & 0,623 & 0,566 & 0,562 & 0,554 & 0,492 & $\mathbf{1 , 0}$ & 0,429 & 0,876 \\
8. DFH & 0,388 & 0,437 & 0,381 & 0,468 & 0,446 & 0,349 & 0,381 & $\mathbf{1 , 0}$ & 0,819 \\
\hline
\end{tabular}

Nota. As correlações na amostra feminina encontram-se na diagonal superior. As correlações na amostra masculina encontram-se na diagonal inferior:

A matriz de correlações foi submetida a uma análise de eixos principais (PAF) para cada sexo extraindo-se o primeiro fator não rotado. $\mathrm{Na}$ amostra feminina o índice KMO foi de 0,915. Para a amostra masculina o índice KMO foi de 0,917. Os testes Bartlett foram significativos para as duas amostras $(p=0,000)$. Os índices indicavam, portanto, a pertinência da fatorização. Calculou-se, logo a seguir, o índice de congruência $\left(r_{\mathrm{c}}\right)$ entre os fatores $g$ dos dois sexos. $\mathrm{O} r$ foi de 0,99 , significando, portanto, que os fatores $g$ de ambos sexos eram praticamente idênticos. Logo, calculou-se a coluna do fator $g$ (primeiro vetor) utilizando a média dos fatores $\mathrm{g}$ corrigidos pelo índice de precisão de ambos os sexos (Tabela 6).

Tabela 6

Cargas g para Cada Sexo e Vetor g (média)

\begin{tabular}{|c|c|c|c|c|c|c|c|c|}
\hline Testes & $r_{x x}$ & $\begin{array}{l}\text { Raiz quadrada } \\
\qquad r_{x x}\end{array}$ & $\begin{array}{l}\text { Masculino } \\
\text { (carga } g \text { ) }\end{array}$ & $\begin{array}{l}\text { Feminino } \\
\text { (carga } g \text { ) }\end{array}$ & $\begin{array}{c}\text { Média } \\
g\end{array}$ & $d$ & $\begin{array}{c}\text { Média de } g \\
\text { corrigido }\end{array}$ & $\begin{array}{l}\text { Média de } d \\
\text { corrigido }\end{array}$ \\
\hline Informação & 0,886 & 0,941 & 0,878 & 0,845 & 0,862 & 0,211 & 0,915 & 0,224 \\
\hline Semelhanças & 0,869 & 0,932 & 0,854 & 0,872 & 0,863 & $-0,152$ & 0,926 & $-0,163$ \\
\hline Aritmética & 0,815 & 0,903 & 0,779 & 0,804 & 0,792 & 0,064 & 0,877 & 0,071 \\
\hline Vocabulário & 0,869 & 0,932 & 0,850 & 0,832 & 0,841 & 0,104 & 0,902 & 0,112 \\
\hline Compreensão & 0,802 & 0,896 & 0,771 & 0,764 & 0,768 & $-0,036$ & 0,857 & $-0,040$ \\
\hline Dígitos & 0,800 & 0,894 & 0,601 & 0,532 & 0,568 & $-0,145$ & 0,635 & $-0,162$ \\
\hline Raven Colorido & 0,876 & 0,936 & 0,714 & 0,721 & 0,718 & 0,093 & 0,767 & o,099 \\
\hline $\mathrm{DFH}$ & 0,819 & 0,905 & 0,520 & 0,559 & 0,540 & $-0,135$ & 0,597 & $-0,149$ \\
\hline Eigenvalue & & & 4,95 & 4,90 & & & & \\
\hline Variância (\%) & & & 61,88 & 61,274 & & & & \\
\hline$N$ & & & 171 & 164 & & & & \\
\hline
\end{tabular}

O primeiro vetor (coluna do fator $g$ ) foi correlacionado às diferenças de média padronizadas (índices $d$ ) entre ambos os sexos (segundo vetor). A correlação de Spearman apontou índice de 0,24 (valor- $p=0,57$ ) e a correlação de Pearson foi 0,55 (valor- $p=0,16$ ). Tais resultados indicam não haver diferenças significativas de inteligência geral entre os sexos.

\section{Discussão}

A discussão que se segue centralizar-se-á em dois pontos de interesse de acordo com os principais resultados alcançados. O primeiro ponto diz respeito aos resultados de diferenças de sexo em testes de habilidades específicas. Quando isto ocorre, se observa que a maior parte das diferenças cognitivas não é estatisticamente significativa.

Embora no DFH, no qual as meninas obtêm melhor desempenho do que os meninos em todas as faixas etárias, ou seja, dos cinco aos 11 anos de idade, apenas a diferença encontrada entre os cinco e os sete anos (valor $d=0,53$ ou 8 pontos de QI) foi estatisticamente significativa (0,05).

No teste Bender, um instrumento que avalia o nível de desenvolvimento viso-motor, os resultados mostram que as meninas de 5 e 7 anos de idade, de forma semelhante ao DFH, saíram-se significativamente melhor que os meninos (d=-0,224). Segundo Cunha (2002), a maturação 
psicomotora ocorre primeiro nas meninas até os 9-10 anos de idade, posteriormente serão os meninos os que apresentarão vantagem psicomotora. Os dados do presente estudo corroboram tal pressuposto.

No caso do TDE, os resultados encontrados fornecem evidência a favor do postulado que assevera a existência de diferenças nas habilidades acadêmicas entre os sexos (Halpern, 1996, 1997). Assim como os demais autores anteriormente citados, no presente estudo, as meninas obtiveram melhor desempenho do que os meninos, em todas as séries, nos subtestes de escrita e leitura, ambos envolvendo habilidades de memória verbal e processamento de informações fonológicas e semânticas. A diferença significativa do ponto de vista estatístico recaiu na escrita entre a $5^{\circ}$ e $6^{\circ}$ série escolar. Já no subteste de aritmética, o qual requer a capacidade de raciocínio matemático, os meninos obtiveram melhor desempenho, da $1^{\text {a }}$ a $6^{a}$ série. Contudo, nenhuma dessas diferenças foi estatisticamente significativa.

Os dados advindos do R-2 apontam uma tendência de diferenças favoráveis às meninas em todas as faixas etárias estudadas, mas também nenhuma delas foi estatisticamente significativa.

$\mathrm{Na}$ amostra gaúcha, de forma semelhante à amostra mineira, tampouco se observaram diferenças cognitivas significativas relacionadas ao gênero em nenhuma das faixas etárias estudadas.

Em síntese, pode-se afirmar que dos 25 índices $d$ levantados em populações escolares de dois estados brasileiros, encontraram-se apenas três valores significativos, sendo esses valores relacionados a diferenças no DFH e Bender, favoráveis às meninas entre cinco e sete anos de idade, e ao desempenho na escrita, também favorável às meninas na $5^{\circ}$ e $6^{\circ}$ série.

O segundo ponto de discussão é e aplicação do MVC a resultados de sujeitos em uma bateria de testes cognitivos. O MVC é a técnica conveniente para verificar se as diferenças entre homens e mulheres são devidas ao fator $g$ (ou inteligência geral). No presente estudo, conforme se viu anteriormente, as diferenças de gênero em inteligência geral também foram negligenciáveis.

Embora a presente investigação precise ser estendida a amostras de outros Estados brasileiros, em que tenha se aplicado uma bateria com maior diversidade de testes cognitivos que a utilizada, os resultados, contudo, são bastante incisivos. As tênues diferenças de sexo observadas em testes individuais parecem dever-se, em parte, à atuação de fatores físicos específicos relacionados à maturação biológica na infância (fatores que favorecem o desempenho das meninas nos primeiros anos da infância e os meninos na pré-adolescência) e, em parte, às diferenças em fatores cognitivos específicos que agem de forma expressiva, conforme a literatura especializada, no desempenho escolar. Esta última tendência parece manifestar-se de forma sistemática. Ocorre tanto em países ocidentais quanto em países não ocidentais (Abdel-Khalek \& Lynn, 2006). De forma geral, mantém-se a assertiva de não haver diferenças cognitivas de sexo no que se refere à inteligência geral. Resta saber, contudo, o que ocorre em amostras de adultos, tanto no que se refere a testes individuais (habilidades específicas) quanto em uma bateria de testes cognitivos que permita extrair a inteligência geral.

Se houver diferenças cognitivas de sexo não significa dizer que há preponderância de um sobre o outro. O problema não está na constatação das diferenças, mas nos valores segregatórios dados por determinada sociedade a um traço em detrimento do outro. Segundo Halpern (1997), os estereótipos não resultam das pesquisas. Eles são ensinados e aprendidos através da experiência em sociedade. A maneira certa para identificar o que são estereótipos e o que são fatos, assim como orientar ações sociais de compensação é a utilização da investigação sistemática. Nesse sentido, o presente estudo, afirma que, pelo menos entre os cinco e os 11 anos de idade, parece não haver diferenças cognitivas de sexo entre crianças escolares das cidades de Belo Horizonte e Porto Alegre.

\section{Referências}

Abdel-Khalek, A. M., \& Lynn, R. (2006). Sex differences on the Standard Progressive Matrices and in educational attainment in Kuwait. Personality and Individual Differences, 40, 175-182.

Aluja-Fabregat, A., Colom, R., Abad, F., \& Juan-Espinosa, M. (2000). Sex diferences in general intelligence defined as $\mathrm{g}$ among young adolescents. Personality and Individual Differences, $28,813-820$.

Angelini, A. L., Alves, I. C. B., Custódio, E. M., Duarte, W. F., \& Duarte, J. L. M. (1999). Padronização brasileira das Matrizes Progressivas Coloridas de Raven. In J. C. Raven. Manual Matrizes Progressivas Coloridas de Raven: Escala especial. São Paulo, SP: Centro Editor de Testes e Pesquisas em Psicologia.

Codorniu-Roga, M. J., \& Vigil-Colet, A. (2003). Sex differences in psychometric and chronometric measures of intelligence among young adolescents. Personality and Individual Differences, 35, 681-689.

Colom, R. \& Flores-Mendoza, C. E. (2007). Intelligence predicts scholastic achievement irrespective of SES factors: Evidence from Brazil. Intelligence, 35, 243-251.

Colom, R., \& Lynn, R. (2004). Testing the developmental theory of sex diferences inintelligence on 12-18 year olds. Personality and Individual Differences, 36, 75-82.

Colom, R., Juan-Espinosa, M., Abad, F. J., \& García, L. F. (2000). Negligible sex differences in general intelligence. Intelligence, 28(1), 57-68

Cunha, J. A. (2002). Bender na criança e no adolescente. In J. A. Cunha (Ed.), Psicodiagnóstico-V (5. ed., pp. 295-3 16). Porto Alegre, RS: Artmed.

Figueiredo, V. L. M. (2002). Padronização brasileira das Escalas Wechsler de Inteligência para Crianças, Terceira Edição WISC-III. In D. Wechsler. WISC-III: Escala de Inteligência para Crianças: Manual. São Paulo, SP: Casa do Psicólogo.

Halpern, D. (1996). Public policy implications of sex differences in cognitive abilities. Psychology, Public Policy and Law, 2, 56 1574 .

Halpern, D. (1997). Sex differences in intelligence: Implications for education. American Psychologist, 52(10), 1091-1102.

Jensen, A. R. (1998). The g factor. London: Praeger.

Koppitz, E. (1989). O Teste Guestáltico Bender para Crianças. Porto Alegre, RS: Artes Médicas. 
Lubinsk, D. (2004). Introduction to the special section on cognitive abilities: 100 Years After Spearman's - 1904. '«General Intelligence', Objectively Determined and Measured». Journal of Personality and Social Psychology, 86(1), 96-111.

Lynn, R. (1999). Sex differences in intelligence and brain size: A developmental theory. intelligence, 27(1), 1-12.

Lynn, R. (2002). Sex differences on the progressive matrices among 15-16 year olds: Some data from South Africa. Personality and Individual Differences, 33, 669-673.

Lynn, R., Allik, J., Pullmann, H., \& Laidra, K. (2004). Sex diferences on the progressive matrices among adolescents: Some data from Estonia. Personality and Individual Differences, $36,1249-1255$.

Lynn, R., Backhoff, E., \& Contreras-Niño, L. A. (2004). Sex diferences on $\mathrm{g}$, reasoning and visualisation tested by the progressive matrices among 7-10 year olds: Some normative data for Mexico. Personality and Individual Differences, 36, 779787.

Lynn, R., Fergusson, D. M., \& Horwood, L. J. (2005). Sex differences on the WISC-R in New Zealand. Personality and Individual Differences, 39, 103-114.

Lynn, R., \& Irwing, P. (2004). Sex differences on the progressive matrices: A meta-analysis. Intelligence, 32, 481-498.

Lynn, R., Raine, T. A., Venables, P. H., Mednick, S. A., \& Irwing, P. (2005). Sex differences on the WISC-R in Mauritius. Intelligence, 33, 527-533.

Mackintosh, N. J. (1996). Sex differences and IQ. Journal of Biosocial Science, 28, 559-572.

Neubauer, A. C., \& Fink, A. (2003). Fluid intelligence and neural efficiency: Effects of task complexity and sex. Personality and Individual Differences, 35, 811-827.

Nyborg, H. (2005). Sex-related differences in general intelligence $\mathrm{g}$, brain size, and social status. Personality and Individual Differences, 39, 497-509.

Oliveira, R., Rosa, H. R., \& Alves I. C. B. (2000). R-2. Teste não verbal de inteligência para crianças: Manual. São Paulo, SP: Vetor.

Stein, L. M. (1994). TDE: Teste de desempenho escolar: Manual para aplicação e interpretação. São Paulo, SP: Casa do Psicólogo.

Wechsler, S. (2003). DFH III: O desenho da figura humana: Avaliação do desenvolvimento cognitivo de crianças brasileiras (3. ed.). Campinas, SP: LAMP/PUC. 